\title{
Evaluation socio-économique d'un projet d'irrigation et étude prospective de la demande en eau
}

\author{
Thierry Rieu - Guy Gleyses \\ CEMAGREF 361, rue J.-F. Breton, B.P. 5095 \\ 34033 Montpellier \\ Tél. 67046300
}

\section{Introduction}

De nombreux travaux, présentés lors des Assises Nationales de l'Eau, posent, comme première condition à une bonne gestion de la ressource en eau, l'adéquation entre ressource et demande en eau. En effet lorsque cet ajustement ne se réalise pas, des déséquilibres se traduisant soit par des tensions sur l'usage de l'eau, soit par une sousutilisation des aménagements, apparaissent et sont coûteux pour la collectivité en termes économiques et écologiques.

Parmi les différents instruments à mettre en œuvre pour faciliter ces ajustements, il semble important d'améliorer les travaux de planification et notamment de s'appuyer sur une meilleure connaissance de la demande en eau agricole.

Les travaux décrits dans la présente communication répondent à cette problématique en proposant une méthodologie dont le premier résultat est l'évaluation de la demande en eau agricole à court et moyen terme.

Les études économiques préalables au projet d'irrigation du Nord-Sommiérois (Gard), conduites en étroite collaboration entre la Compagnie du Bas-Rhône et du Languedoc (BRL) et le Centre National du Machinisme Agricole, du Génie Rural, des Eaux et Forêts (CEMAGREF), servent de support à la présentation de la démarche.

\section{Présentation de la région étudiée}

Située à la limite de la plaine languedocienne et des premiers contreforts des Cévennes, la petite région du Nord-Sommiérois regroupe un ensemble de onze communes ayant manifesté la volonté conjointe de s'engager dans un projet d'irrigation.

Par le passé, plusieurs tentatives de réalisation d'un périmètre irrigué ont échoué du fait d'une demande en eau trop faible expliquée par une orientation viticole quasi exclusive.

Actuellement, la demande d'irrigation est plus ferme et est complétée par des besoins exprimés par les communes (eau potable, lutte contre les feux de forêts...).

La petite région présente un paysage homogène, vallonné et s'étageant entre des altitudes de 25 à $200 \mathrm{~m}$, dans lequel s'insèrent les terrasses du Vidourle et de ses petits affluents. C'est sur ces terrasses que s'établit l'activité agricole, les hauteurs étant occupées par la garrigue ou des bois.

Le climat, méditerranéen, se caractérise par des précipitations violentes en automne et au printemps, des étés chauds et secs, et conduit à un déficit hydrique important.

Economic study of an irrigation scheme and evaluation of the medium run agricultural water demand

In the context of economical pre-feasibility study for the design of an irrigation system, CEMAGREF has developed a methodology and carried it out in the Nord-Sommierois region (Gard-South of France). Its main claim is to evaluate the medium run agricultural water demand in order to fit water requirements, at the scheme scale, and water ressources (supply). The methodology is based on the analysis of farm management, focusing on agricultural supply modelisation, using linear programming. Simulations are implemented with different scenarios of changes in the economical and irrigation context. Results are given for two scenarios. This methodology can be considered as a decision support tool for the technical and financial design of irrigation systems. Its use should be restricted to pre-feasibility studies on small areas (around 200 farms). 
Le paysage agricole est largement dominé par la présence de la vigne. La production viticole est encore essentiellement composée de vin de qualité courante, mais plusieurs coopératives améliorent leur vignoble et obtiennent le classement d'une partie du terroir en Apellation d'Origine Contrôlée.

Depuis ces dernières années, les cultures arboricoles et légumières suscitent un intérêt croissant qui se traduit par leur développement à proximité du Vidourle, seule ressource en eau disponible. Cependant le débit d'étiage du fleuve est particulièrement faible, inférieur certaines années à $50 \mathrm{l} / \mathrm{s}$ à l'aval de Sommières.

Les infrastructures de BRL s'arrêtent à la limite du périmètre avec une conduite d'un diamètre de $900 \mathrm{~mm}$ qui est, dans cette région, la partie terminale de l'adduction depuis le Rhône.

\section{Modèle économique de prévision de la demande en eau}

\subsection{Méthodologie}

La méthodologie développée par le CEMAGREF évalue l'impact d'un projet d'irrigation sur la production agricole de la zone concernée par l'aménagement. Elle a pour objectif de localiser, de prévoir à court et moyen terme, la demande en eau agricole ainsi que de fournir une analyse de l'utilisation agricole de l'espace.

La quantification de la demande agricole permet de dimensionner le réseau pour satisfaire les besoins de pointe, de rechercher les ressources en eau les mieux adaptées et enfin de réaliser une analyse financière de l'investissement.

La localisation de la demande en eau aide à choisir les surfaces à irriguer et est à la base du tracé du réseau.

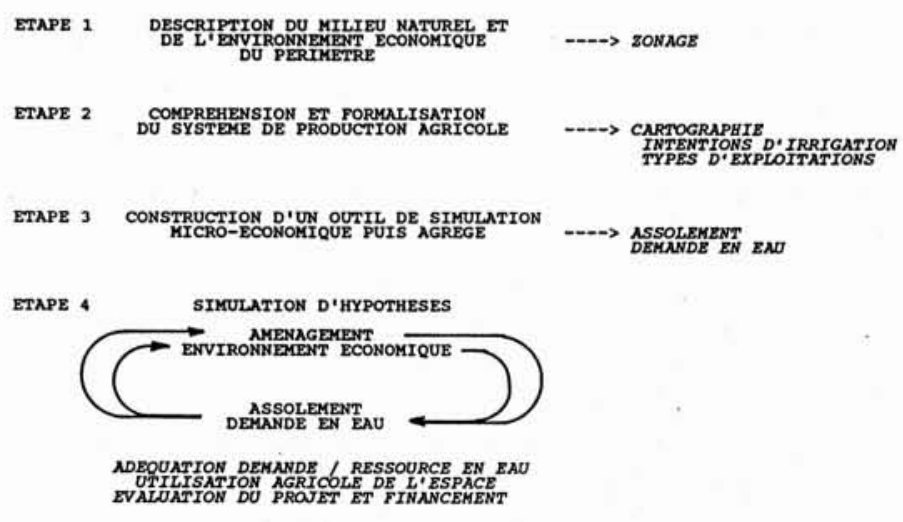

1. Démarche d'étude.
Sous l'effet du renouvellement des exploitations et de l'évolution de l'environnement économique, la demande en eau évolue au fil des années.

A court terme, les déclarations des agriculteurs fournissent une évaluation de la demande souvent surestimée.

A moyen terme, les prévisions sont plus difficiles compte tenu de la diversité des systèmes de production et de l'évolution complexe de l'environnement économique.

Dans les deux cas, la modélisation de l'offre agricole à l'échelle du périmètre irrigué est utilisée pour valider les intentions des agriculteurs et pour fournir une analyse prospective à moyen terme de la demande en eau agricole.

La formalisation économique adoptée relève du cadre général de la théorie micro-économique et suppose que les choix des agriculteurs sont rationnels compte tenu des contraintes qui pèsent sur leur exploitation. Deux hypothèses complémentaires traduisent la spécificité de la démarche :

- compte tenu de l'hétérogénéité des exploitations, il est probable que les réactions des exploitants, face au contexte nouveau créé par l'opportunité d'irriguer, soient ellesmêmes diversifiées. Le modèle agrégé rend compte de cette diversité de comportement face à l'irrigation en distinguant plusieurs types d'exploitation et en modélisant leur fonctionnement ;

- en considérant que des facteurs de production comme la disponibilité en terre ou la main-d'œuvre permanente, par exemple, sont fixes ou connaissent une évolution prévisible sous l'effet du renouvellement des exploitations.

Le choix de cette formalisation induit aussi des limites, notamment du fait de l'utilisation de modèles linéaires et déterministes qui rendent mal compte de l'attitude des agriculteurs vis-à-vis des risques climatiques et économiques.

Du fait de l'enquête exhaustive des exploitations comprises dans la zone d'étude, la méthode demeure réservée aux études préalables de petits périmètres (environ 100 à 200 exploitations soit 1000 à 3000 ha).

\subsection{Démarche utilisée}

Quatre étapes principales peuvent être distinguées dans la démarche (cf. fig. 1).

La première étape a pour objectif d'améliorer la connaissance de la zone et de réaliser un découpage du périmètre en sous-ensembles, sur la base de données physiques (climat, sols, potentialités agronomiques) et socioéconomiques (structures agraires, Recensement Général Agricole, aménagements déjà réalisés...). Chaque sousensemble présente une homogénéité tant des potentialités agronomiques que des cultures possibles. A Sommières, six îlots de cultures sont délimités en fonction des contraintes pédologiques les plus importantes (profondeur et pierrosité) et de la présence de vignes de moins de 25 ans ayant la plus forte proportion de cépages améliorateurs.

La seconde étape, essentielle pour la modélisation microéconomique, a pour objectif de comprendre et de formaliser le système de production agricole. 
La typologie des pratiques culturales et des exploitations est réalisée à partir des exploitations recensées sur deux groupes de communes:

- Sept communes où existe un projet d'équipement hydraulique.

- Deux communes voisines équipées d'un réseau d'irrigation depuis plus de 10 ans. Leurs exploitations servent de références pour les itinéraires techniques et les systèmes de production en situation irriguée.

Les pratiques culturales sont construites à partir du calendrier des opérations observées sur les parcelles de chaque exploitation. Dans ce processus, ne sont retenues que les opérations qui sont significatives en terme de contraintes ou d'objectifs de l'exploitant (temps et calendrier de travail, contraintes pédologiques, marge brute, risques encourus...). Elles rendent compte des techniques de production employées et du degré d'innovation technique.

Compte tenu de l'hétérogénéité des exploitations, il est probable que les réactions des exploitants, face au contexte nouveau créé par l'opportunité d'irriguer, soient ellesmêmes diversifiées. Pour une analyse au niveau régional, il est nécessaire d'agréger les exploitations afin d'obtenir une vision d'ensemble de l'évolution potentielle de l'agriculture. Cette agrégation est réalisée sur la base des réactions attendues des agriculteurs ou, en d'autres termes, sur leur type de comportement. En supposant que les comportements sont essentiellement déterminés par les stratégies des exploitants, l'analyse des systèmes de production permet alors d'identifier ces stratégies et de regrouper les exploitations en classes homogènes par rapport à ce critère.

La construction de la typologie des exploitations tient compte simultanément des structures (main d'œuvre, surfaces de différentes potentialités agronomiques, âge de l'exploitant et composition de la famille) et des pratiques culturales dominantes révélatrices des orientations de l'exploitation (cf. fig. 2).

A titre d'exemple, les exploitations du type $2 \mathrm{~F}$ sont engagées dans une stratégie de dynamique viticole avec des cultures de diversification. Elles se caractérisent par une surface agricole et des disponibilités en main d'œuvre importantes (un travailleur pour 13 hectares). Cela leur permet d'avoir une activité viticole modernisée comparable à celle du type $1 \mathrm{~F}$ et d'envisager des cultures irriguées intensives, arboriculture, raisin de table, maraîchage de plein champ. Les grandes cultures irriguées, tel le tournesol ou le maïs grain, peu exigeantes en travail sont absentes de l'assolement.

La troisième étape correspond à la construction d'un modèle agrégé à l'échelle du périmètre.

Au préalable, un modèle est construit pour chaque type d'exploitation. Le modèle explique les choix d'assolement des exploitations, en fonction des disponibilités en terre, en main d'œuvre permanente, en eau, en droits de plantation... pour obtenir le revenu d'exploitation maximum, sous l'hypothèse de rationalité des choix des agriculteurs.

C'est un modèle d'optimisation linéaire dont le programme s'écrit :

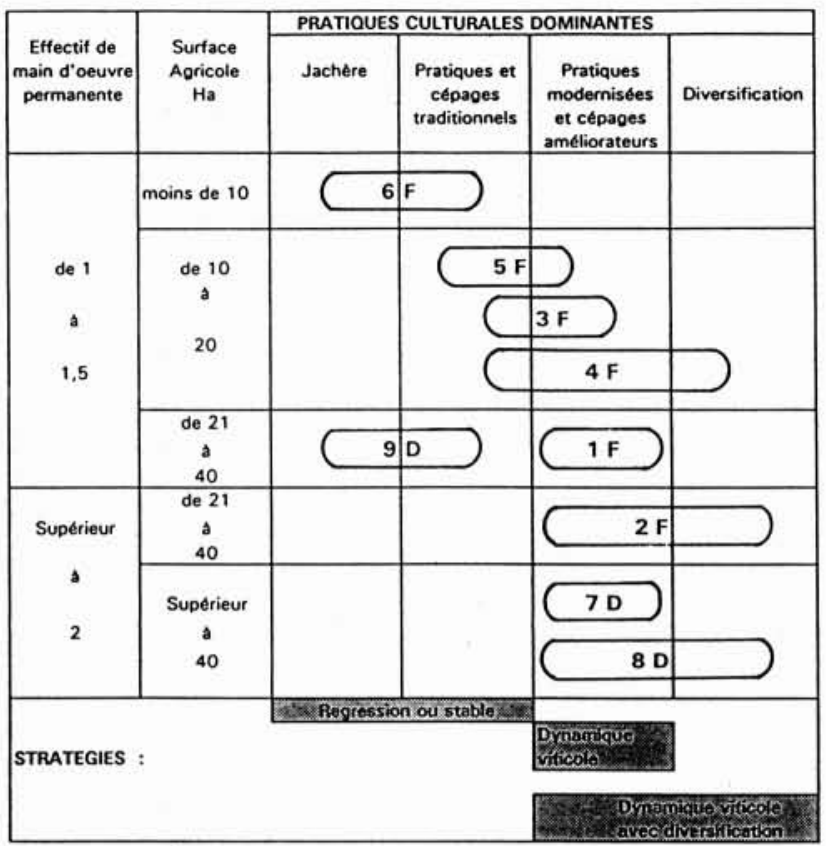

Légende

1F : Type 1, exploitations familiales

7D : Type 7, exploitations de domaines

2. Présentation de la typologie des 138 exploitations du périmètre (9 types).

Maximiser $C^{\prime} X$, sous les contraintes :

$A X \leqslant B$ et $X \geqslant 0$

où $X=(x j), x j$ étant la surface attribuée à la culture $j$. $A=(a i j)$, est la matrice des coefficients techniques où chaque élément aij est la quantité de ressource $i$ nécessaire pour produire une unité de la culture $j$.

$B=(b i), b i$ est la quantité de ressource $i$ disponible.

$C=(c j), c j$ est la marge unitaire de l'activité $j$.

Les ressources $b i$ sont estimées par agrégation des données recueillies lors des enquêtes exhaustives d'exploitation.

Les marges $c j$ et les coefficients techniques aij sont estimés à partir d'hypothèses de production, de charges, de quantité de travail à l'hectare et sur la base des résultats d'enquêtes.

Cela conduit parfois à décrire plusieurs itinéraires techniques pour une même culture lorsque, par exemple, la main d'œuvre pour la récolte des asperges peut être saisonnière ou familiale.

Un calage du modèle est réalisé par ajustement des paramètres, d'abord pour les modèles de chaque type d'exploitation (ajustement des $c j$ et $a i j$ ), ensuite pour le modèle agrégé (ajustement des bi).

Le modèle est considéré comme calé lorsqu'il permet de retrouver la structure de l'assolement observé et qu'il rend bien compte du comportement de chaque type d'exploitation. 
3.

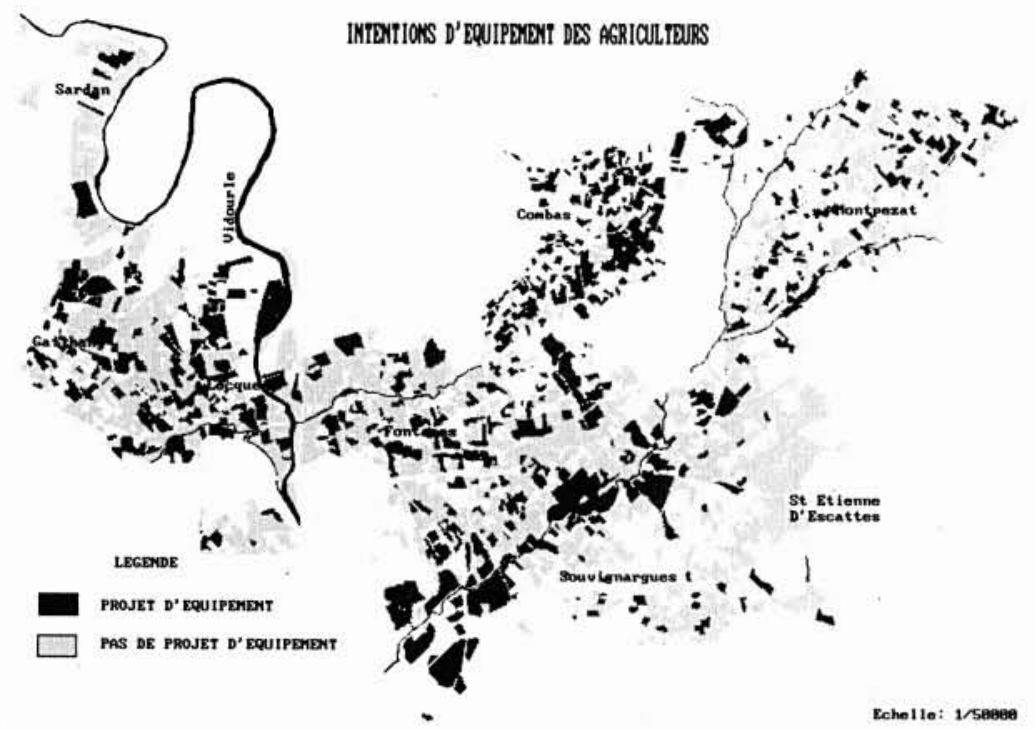

4.

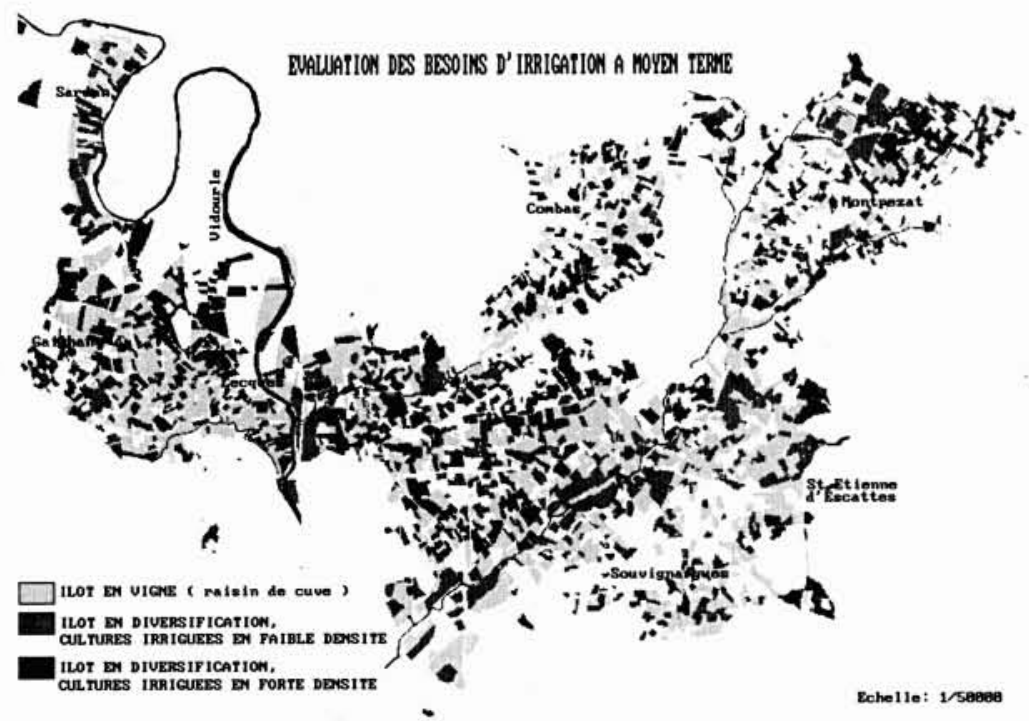

Echelle: 1/125000

Tableau 1. Assolement calculé pour un équipement de tout le périmètre.

\begin{tabular}{|l|c|}
\hline Cultures & Surface (hectares) \\
\hline Melon & 130 \\
Asperge & 159 \\
Raisin de table & 154 \\
Arboriculture & 34 \\
\hline Total cultures irriguées & 477 \\
\hline Vigne (cuve) & 1736 \\
Céreales & 282 \\
\hline Surface cultivee totale & 2495 \\
Jacherres & 201 \\
\hline Surface totale & 2696 \\
\hline
\end{tabular}

Tableau 2. Evolution de l'assolement en fonction de la marge brute du raisin de table.

\begin{tabular}{|l|r|r|r|r|}
\hline $\begin{array}{l}\text { Marge brute du raisin } \\
\text { de table (F/ha) }\end{array}$ & 26000 & 24000 & 22000 & 20000 \\
\hline Melon & 130 & 132 & 131 & 131 \\
Asperge & 159 & 159 & 162 & 174 \\
Raisin de table & 154 & 150 & 123 & 66 \\
Arboriculture & 34 & 34 & 34 & 34 \\
\hline Total cultures irrigutes & 477 & 475 & 450 & 405 \\
\hline Vignes (Cuve) & 1736 & 1738 & 1769 & 1817 \\
Céreales & 283 & 285 & 282 & 281 \\
\hline Jachères & 201 & 198 & 195 & 193 \\
\hline Surface totale & 2696 & 2696 & 2696 & 2696 \\
\hline
\end{tabular}

Tableau 3. Comparaison des assolements calculés en 1991 et en 1998

\begin{tabular}{|l|r|r|}
\hline Annfé de la situation foncière & 1991 & 1998 \\
\hline Melon & 130 & 118 \\
Asperge & 159 & 161 \\
Raisin de table & 154 & 171 \\
Arboriculture & 34 & 33 \\
\hline Total cultures irriquées & 477 & 483 \\
\hline Vigne (cuve) & 1736 & 1761 \\
Céréales & 282 & 304 \\
\hline Surface cultivée & 2495 & 2548 \\
\hline
\end{tabular}


Toutefois, après validation, des différences peuvent subsister, par exemple les surfaces calculées en céréales sont en valeur absolue différentes de la réalité. Cet écart s'explique par les contraintes d'accessibilité des parcelles qui ne sont pas prises en compte dans le modèle et par le caractère moins risqué de la culture de blé par rapport à celle des cultures irriguées.

Dans la quatrième étape, le modèle est utilisé pour tester des scénarios portant sur :

- l'évolution de l'environnement économique ;

- le renouvellement des exploitations ;

- les modalités d'aménagement.

Cette phase importante est illustrée par les résultats fournis dans le chapitre suivant.

\section{Résultats}

Les résultats obtenus proviennent aussi bien de l'utilisation des données d'enquête que de la modélisation.

\section{I. Les résultats des enquêtes d'intention d'équiper}

Les intentions des agriculteurs d'équiper leurs parcelles concernent 960 ha soit $36 \%$ de la surface enquêtée. Cette demande a deux caractéristiques :

- elle concerne une part significative de vignes (production de vin) dont les besoins d'irrigation sont faibles;

- la cartographie montre une demande peu concentrée (cf. fig. 3).

\subsection{Les résultats de simulations à l'aide du modèle agrégé}

Le premier scénario correspond à l'équipement de la totalité du périmètre.

\subsubsection{L'assolement calculé}

Dans le contexte pédo-climatique de Sommières l'irrigation de la vigne nécessite un ou deux arrosages par an et reste aléatoire. Il a été décidé de ne pas modéliser cette pratique.

Le tableau 1 présente l'assolement calculé pour un équipement de tout le périmètre. Le modèle prévoit une superficie irriguée hors vigne de 477 ha. Les principales cultures irriguées sont le melon, l'asperge et le raisin de table. Le modèle prévoit peu de surface avec des abricotiers dans la mesure où ceux-ci sont exigeants en main-d'œuvre permanente pour l'éclaircissage des fruits.

Les grandes cultures irriguées ne sont pas sélectionnées par le modèle en raison de l'importance du coût de l'eau comparé à la marge brute des cultures.

Les surfaces irriguées prévues à court terme par le modèle, 477 ha, sont inférieures de $13 \%$ aux projets des agriculteurs pour irriguer des cultures autres que la vigne.
Cette différence n'est pas très élevée, de plus elle est due à la présence des cultures céréalières et industrielles dans les projets des agriculteurs alors que le modèle ne les choisit pas.

Dans les conditions actuelles, en disponibilités de main d'œuvre permanente et de surface, de prix et de rendements des cultures, le calcul économique montre que les intentions d'irrigation des agriculteurs sont assez réalistes.

Les tests de sensibilité permettent d'une part d'analyser la stabilité du modèle et d'autre part d'apprécier l'évolution des surfaces des différentes cultures (irriguées ou non) lorsque le système de prix varie.

La stabilité du modèle est testée en faisant varier les prix de vente des produits de la vigne qui est la principale activité des exploitations.

Le modèle est assez stable puisqu'une baisse de $15 \%$ des prix se traduit par une diminution de la surface en vigne de $4 \%$; la surface en culture irriguée augmente de $7 \%$ en passant de 477 à 510 ha.

Pour le raisin de table par exemple, il faut une diminution de $6000 \mathrm{~F} /$ ha de la marge brute (passage de $26000 \mathrm{~F} /$ ha à $20000 \mathrm{~F} / \mathrm{ha}$ ) pour obtenir une forte réduction des surfaces. Les surfaces perdues en raisin de table se reportent partiellement sur les asperges. Les surfaces en melon et en vergers restent inchangées (cf. tabl. 2). Au total la surface des cultures irriguées diminue de $15 \%$.

Pour une projection à moyen terme, il est nécessaire de tenir compte de l'évolution foncière et du renouvellement des exploitations. Ainsi 269 hectares de terres sont disponibles sur le marché foncier local du fait de la disparition d'exploitations tenues par des agriculteurs âgés et sans succession. (Type 6 par exemple.)

Les terres libérées sont redistribuées sur la base des projets fonciers des agriculteurs et de l'analyse de la valeur marginale de la terre.

Le modèle prévoit une surface cultivée totale de 2548 ha sur l'ensemble du périmètre; soit une augmentation de 53 ha. Les surfaces en céréales et vignes ont la plus forte augmentation, respectivement 22 et 25 ha, la surface irriguée augmente de 6 ha (cf. $t a b l .3$ ).

La surface irriguée reste stable, les changements les plus importants portent sur le choix des espèces : la surface en melon régresse au profit de celle en raisin de table.

\subsubsection{La localisation de la demande}

Pour accroitre la précision de la localisation de la demande, le périmètre a été découpé en sept secteurs. Chacun de ces secteurs a été étudié séparément à l'aide d'un modèle agrégé.

La part des surfaces irriguées par rapport à la surface totale la plus élevée est localisée sur le secteur de Combas $(27 \%)$, elle varie de 18 à $21 \%$ sur les secteurs de Gailhan, Fontanes, Lecques et Sardan, enfin elle est plus faible sur les secteurs de Montpezat et de Souvignargues (respectivement 14 et $9 \%$ ).

Cette demande est cartographiée selon deux modes de densité d'irrigation (cf. fig. 4). 


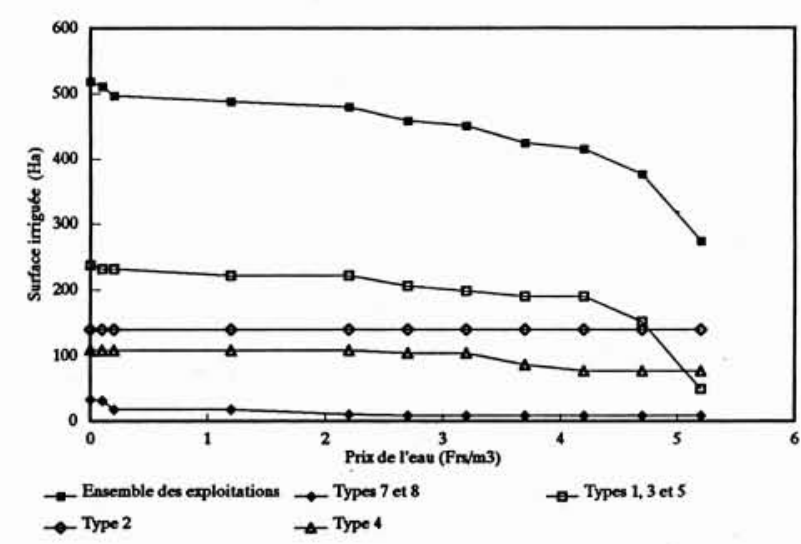

5. Fonction de demande en eau. (Demande globale et par type d'exploitation.)

\subsubsection{Fonction de demande en eau}

La demande en eau est calculée pour la durée de la campagne agricole, sur la base des assolements prévus par le modèle et des besoins en eau calculés en année quinquennale sèche.

Le débit fictif tient compte des pratiques locales d'irrigation et est calculé sur la base d'une durée journalière d'arrosage de 18 heures. Le débit fictif du périmètre pour le mois de pointe est environ de $600 \mathrm{l} / \mathrm{s}$ pour 1000 ha irrigués.

Dans le cas du Nord-Sommiérois, le prix de l'eau est fixé par la tarification uniforme mise en place sur l'ensemble du département du Gard. Néanmoins, même si le calcul d'un prix de l'eau ne se pose pas, il demeure intéressant d'observer la sensibilité de la demande au prix de l'eau.

La fonction de demande (fig. 5) révèle une faible élasticité de la demande. Ceci est dû au choix par le modèle de cultures irriguées à fortes marges et où le prix de l'eau représente une faible part (de l'ordre de 2 à $4 \%$ ) des charges totales.

Une analyse des fonctions de demande par type d'exploitation montre des sensibilités différentes : les types 7 et 8 sélectionnent des grandes cultures irriguées lorsque le prix de l'eau est inférieur à $0,30 \mathrm{~F} / \mathrm{m}^{3}$. Le type 2 qui diversifie en choisissant l'arboriculture et la culture du melon est insensible au prix dans la plage envisagée. Enfin les types 1,3 et 5 qui pratiquent une diversification basée sur les asperges et le raisin de table, présentent une courbe de demande faiblement décroissante jusqu'à $4 \mathrm{~F} / \mathrm{m}^{3}$, au-delà les surfaces irriguées diminuent fortement.

\subsubsection{L'analyse financière}

Les coûts d'investissement pour la mise en pression et la distribution à la parcelle sont de 58,2 millions de francs dans l'hypothèse d'un équipement de tout le périmètre (3 158 ha).

L'investisssement est subventionné à 70 et $80 \%$ par l'Etat et les collectivités locales selon la nature des équi- pements, soit un montant de subventions de 42,7 millions de francs.

Le bilan d'exploitation du gestionnaire, sur une période de 25 ans, prend en compte :

- les dépenses dues aux charges de remboursement d'emprunt, aux coûts d'entretien et de gestion du périmètre ; - les recettes réalisées sur les ventes d'eau, celles-ci étant évaluées à partir des prévisions de souscription et de tarifications moyennes.

Compte tenu de cette subvention, le bilan cumulé sur 25 ans est largement déficitaire (14,6 millions de francs). Le bilan annuel fait apparaître un excédent à partir de la dix-neuvième année, mais qui est insuffisant pour combler rapidement le déficit créé jusqu'à cette date.

Les résultats s'expliquent par des coûts d'investissement importants pour un réseau très étendu et par la faiblesse des recettes face aux dépenses dues à un poids important des remboursements d'emprunt.

\subsection{Test d'un nouveau scénario d'aménagement}

Les résultats médiocres en terme d'équilibre financier ont conduit à étudier un équipement partiel du périmètre sur les secteurs où la demande en eau est la plus dense.

Dans ce scénario, la surface irrigable s'établit à 1456 ha contre 3158 ha dans le cas d'un équipement total.

Le modèle prévoit 315 ha de cultures irriguées hors vigne: il y a bien concentration des surfaces irriguées puisque le ratio Surface Irriguée/Surface Equipée passe de 15 à $22 \%$. La nature de l'assolement étant peu modifiée, le débit fictif de pointe demeure égal à $0,6 \mathrm{l} / \mathrm{s} / \mathrm{ha}$.

Dans le cadre de cet équipement partiel, la création de deux retenues collinaires permet de mobiliser une grande partie des ressources en eau nécessaires sur les bassins versants de deux affluents du Vidourle. Ces retenues desservent Gailhan et Lecques d'une part et Combas d'autre part ; l'ensemble Fontanes-Souvignargues est alimenté à partir de l'adducteur existant du réseau BRL.

Les coûts d'investissement pour les deux retenues, le réseau d'adduction avec les surpresseurs, et le réseau de distribution à la parcelle s'élève à 38,6 millions de francs.

Le bilan d'exploitation au terme des 25 ans fait toujours apparaître un déficit. Le bilan annuel est excédentaire à partir de la dix huitième année et les déficits des premières années seraient comblés au terme de 45 ans d'exploitation au lieu de 65 ans pour un aménagement global du périmètre.

\section{Bilan}

Tout au long de l'étude, l'application de cette démarche a permis de fournir aux différents partenaires (agriculteurs, maires, Conseil Général) des éléments d'aide à la décision sous forme, soit de données d'enquête sur support cartographique, soit de résultats de modélisation pour différents scénarios d'aménagement. 


\section{ÉVALUATION SOCIO-ÉCONOMIQUE D'UN PROJET}

Tous les scénarios d'aménagement faisant apparaître des comptes prévisionnels d'exploitation déficitaires pendant le remboursement des emprunts, les communes du NordSommiérois se sont portées maître d'ouvrage de l'opération sous forme d'un syndicat intercommunal. Elles ont aussi décidé de participer au financement des investissements. Le Conseil Général du Gard a prévu d'attribuer une subvention d'exploitation d'un montant plafonné.

Pour les communes, le scénario correspondant à un équipement partiel du périmètre ne présente pas d'avantage financier décisif. Elles préfèrent équiper la totalité du périmètre et offrir la possibilité d'irriguer à un plus grand nombre d'agriculteurs.

La mise en place d'une maîtrise d'œuvre partagée est sans précédent dans le département du Gard. Les élus locaux et le gestionnaire en attendent un partage des risques financiers et une meilleure gestion du réseau dans la mesure où les différents utilisateurs sont directement impliqués.

\section{Conclusion}

La méthode présentée et illustrée par les études économiques préalables au projet d'irrigation du NordSommiérois, semble constituer un outil pertinent pour l'évaluation de la demande en eau agricole et sa localisation.

La nature prospective de la modélisation permet, à partir d'hypothèses d'évolution de l'environnement économique et d'aménagement du territoire, de mesurer les effets de différents scénarios sur la demande en eau à moyen terme.

Dans le cadre des études préalables à la conception des réseaux d'irrigation, elle fournit aux différents partenaires des éléments d'aide à la décision.

Toutefois son utilisation demeure limitée à de petits périmètres, dans la mesure où l'analyse du fonctionnement des exploitations agricoles repose sur un système d'enquêtes exhaustives. Cette méthode doit donc être réservée à des périmètres n'excédant pas 200 exploitations afin de la réaliser dans un délai (un an) compatible avec celui des autres études techniques préalables.

A cette échelle, elle demeure reproductible puisqu'elle a été appliquée dans différents contextes de production agricole :

- systèmes de cutlures pérennes, maraîchage de plein champ et grandes cultures dans la plaine Languedocienne ; - systèmes d'élevage bovin et grandes cultures dans le Nord-Est du Massif Central ;

- système d'élevage ovin et maraîchage dans les Alpes du Sud-Est.

\section{Bibliographie}

[1] Long M., Blanc, J.G., Belluau E., Goudeau A., 1991. Fiches SIMAGRI Gard-Hérault. Références technicoéconomiques pour les principales cultures. CNABRL, $138 \mathrm{p}$.

[2] Citeau J.M., Gleyses G., Rieu T., 1992. - Etude économique préalable à la conception d'un réseau d'irrigation et évaluation de la demande en eau. Communication CIID, La Haye (à paraître), 11 p.

[3] Boussard J.M., 1987. - Economie de l'agriculture. Economica, $310 \mathrm{p}$.

[4] Rieu T., Martinand P., Diquelou P., 1989. - Analyse économique préalable à la conception d'un périmètre irrigué dans le canton de St-Auban. Génie Rural, n 12 , pp. 78 à 83 . 